Research article

\title{
Clinical significance of Neutrophil gelatinase-associated lipocalin(NGAL) expression in primary rectal cancer
} Xiu-Feng Zhang1, Ying Zhang'2, Xiao-Hua Zhang*1, Su-Mei Zhou1, GuanGen Yang ${ }^{3}$, Ou-Chen Wang1, Gui-Long Guo ${ }^{1}$, Gao-Yi Yang ${ }^{2}$ and Xiao-Qu Hu${ }^{1}$

Address: ${ }^{1}$ Department of Oncological Surgery, the First Affiliated Hospital of Wenzhou Medical College, Wenzhou, 325000, PR China, ${ }^{2}$ Department of Ultrasound, the Red Cross Hospital of Hangzhou, Hangzhou, 310003, PR China and ${ }^{3}$ Department of Colorectal Surgery, the Third People's Hospital of Hangzhou, Hangzhou, 310009, PR China

Email: Xiu-Feng Zhang - legandsky520@163.com; Ying Zhang - lmzy1386@163.com; Xiao-Hua Zhang* - oncology0607@163.com; SuMei Zhou - surzsm@163.com; Guan-Gen Yang -ygg1948@sohu.com; Ou-Chen Wang -woc099@gmail.com; Gui-

Long Guo - guoguilong@sina.com; Gao-Yi Yang - prettysky@yahoo.com.cn; Xiao-Qu Hu - huxiaoqu57@163.com

* Corresponding author

Published: 6 May 2009

BMC Cancer 2009, 9:134 doi:10.1 186/147|-2407-9-134
Received: 16 October 2008

Accepted: 6 May 2009

This article is available from: http://www.biomedcentral.com//47/-2407/9//34

(C) 2009 Zhang et al; licensee BioMed Central Ltd.

This is an Open Access article distributed under the terms of the Creative Commons Attribution License (http://creativecommons.org/licenses/by/2.0), which permits unrestricted use, distribution, and reproduction in any medium, provided the original work is properly cited.

\begin{abstract}
Background: Emerging evidence has demonstrated that Neutrophil gelatinase-associated lipocalin (NGAL) is up-regulated in multiple malignancies, including oesophagus cancer, and plays a critical role in tumorigenesis and progression. However, till now, little is known about the role of NGAL in human rectal cancer. Its association with clinicopathologic characteristics and expression of MMP-9, one of its target genes, has not been reported systematically in rectal cancer. Therefore, to further determine the potential involvement of NGAL in rectal cancer, we have evaluated the expression level of NGAL mRNA by real time RT-PCR, and further elucidated the correlation of NGAL mRNA expression with clinicopathologic features and MMP-9 in rectal cancer.
\end{abstract}

Methods: 100 paired samples of rectal cancer and adjacent normal tissues obtained from inpatients undergoing surgical operation were allocated into two groups (cancer group and control group). The mRNA expression of NGAL and MMP-9 was determined by real-time RT-PCR. The association between their expression and clinicopathological characteristics of rectal cancer were analysised.

Results: Among the 100 rectal cancers, 69 cases of NGAL mRNA up-regulation were observed. NGAL mRNA up-regulation was positively correlated with MMP-9 $\left(r_{\mathrm{s}}=0.393, p<0.00 \mathrm{I}\right)$. In rectal cancer, NGAL mRNA overexpression was significantly associated with depth of invasion $(p=$ $0.028)$, lymph node metastasis $(p=0.009)$, venous involvement $(p=0.023)$ and advanced PTNM stage $(p=0.0 \mathrm{II})$.

Conclusion: In human rectal cancer, NGAL mRNA expression was elevated. NGAL mRNA upregulation was correlated significantly with tumor progression and MMP-9 mRNA overexpression in rectal cancer, suggesting a more aggressive phenotype. NGAL could be used for rectal cancer characterization. 


\section{Background}

Colorectal cancer (CRC) is one of the common gastrointestinal cancers worldwide and the second leading cause of cancer-related deaths in Europe[1]. In the United States, colorectal cancer was the third most common cause of cancer related death in 2007[2]. About 30\% to $60 \%$ of patients with colorectal cancer undergoing primary surgery with curative intention still die from metastatic disease[3]. Compared to colon cancer, rectal cancer, with worse prognosis, is mainly attributed to higher advanced cancer resulting from progression and metastasis of the tumor, which are two of the most important factors in determining the prognosis of patients with rectal cancers[4]. The early diagnosis of rectal cancer is difficult owing to the late presentation of symptom. The scarcity of early biomarkers has considerably hindered our ability to launch preventive measures for this malignancy in a timely manner. So it is important to find superior tumor markers implicated in the progression and metastasis of human rectal cancer.

NGAL, a 24-kDa glycoprotein, also known as lipocalin-2, belongs to the lipocalin protein family. The rat NGAL homologue, neu-related lipocalin (NRL), was first identified as a gene whose expression was specifically induced in HER-2/neu oncogene-induced rat mammary carcinomas[5]. NGAL exists as monomer (25 kDa), homodimer (46 kDa), and disulfide-linked heterodimer with Matrix Metalloproteinase-9 (MMP-9,135 kDa)[6,7]. As a member of the MMP family, MMP-9 has been implicated to cancer progression by degradation of the molecular components of the basement membrane and extracellular matrix, liberating vascular endothelial cell growth factor (VEGF) from the extracellular matrix and therefore enabling angiogenesis, invasion and distant metastasis [810].

Growing evidence has demonstrated that NGAL can form a complex with MMP-9 and improves MMP-9 expression, prevents its degradation, causing increased MMP-9 enzymatic activity, thereby favoring invasive and metastatic potential of cancer cells in vivo and in vitro [11-13].

Elevated NGAL expression has also been detected in a variety of malignancies and involved in the invasion and progression of tumors, including breast cancer, esophageal carcinoma and gastric cancer et al [13-15].

Up to now, however, little is known about the role of NGAL in human rectal cancer. Its association with clinicopathologic characteristics and expression of MMP-9, one of its target genes, in rectal cancer has not been reported systematically. Therefore, to further determine the potential involvement of NGAL in rectal cancer, we detected
mRNA expression of NGAL and MMP-9 in matched rectal samples by real time RT-PCR, and further evaluated the correlation between NGAL and MMP-9 as well as clinicopathological features in human rectal cancer.

\section{Methods \\ Patients and specimens}

100 paired samples of rectal cancer and adjacent normal tissues were obtained from inpatients undergoing surgical operation from January 2006 to September 2008 at the Department of Oncological Surgery, the First Affiliated Hospital of Wenzhou Medical College. These inpatients did not receive any chemotherapy and radiotherapy before operation. This study was approved by the institutional review board and all patients provided written informed consent to participate. The rectal cancer and paired normal rectal tissues were allocated to a cancer and a control group respectively. The adjacent normal tissues were distant from the corresponding rectal cancer tissues more than $5 \mathrm{~cm}$. The samples of rectal cancer and adjacent normal tissues were away from the region of obvious inflammation and necrosis. The immunohistochemistry Staining was routinely performed by the Department of Pathology of the First Affiliated Hospital of Wenzhou Medical College in order to exclude the cases with obvious inflammation and necrosis response, and to reduce the potential interference by neutrophils. All dissected specimens were cut into 5-mm cubic blocks, snap frozen in liquid nitrogen immediately and stored at $-80^{\circ} \mathrm{C}$. Tumor grade of differentiation was defined according to the WHO criteria[16]. The clinical and pathological stages were defined according to pTNM stage. The histopathological examination was conducted by the Department of Pathology of the First Affiliated Hospital of Wenzhou Medical College. As shown in table 1, specific primers and probes for NGAL gene, MMP-9 gene and glyceraldehydes3 phosphate (GAPDH) gene (reference gene) were designed based on sequence data from the Ensembl database http://www.ensembl.org. Primers and probe were placed at the junction between 2 exons. All primers and probes were synthesized by Takara Biotechnology Co., Ltd (Dalian, China).

\section{Real-time RT-PCR}

Real-time RT-PCR was performed using a relative quantification protocol on an iCycler iQ System (Bio-Rad, Hercules, Calif., USA). The optimal conditions for the amplification of NGAL consisted of 2 min at $94^{\circ} \mathrm{C}$ followed by 40 cycles for $30 \mathrm{~s}$ at $94^{\circ} \mathrm{C}, 30 \mathrm{~s}$ at $51^{\circ} \mathrm{C}$ and 30 s at $71^{\circ} \mathrm{C}$. The optimal conditions for the amplification of MMP-9 consisted of 2 min at $94^{\circ} \mathrm{C}$ followed by 40 cycles for $30 \mathrm{~s}$ at $94^{\circ} \mathrm{C}, 30 \mathrm{~s}$ at $56^{\circ} \mathrm{C}$ and $30 \mathrm{~s}$ at $71^{\circ} \mathrm{C}$. In addition, a no-template control (double-distilled $\mathrm{H}_{2} \mathrm{O}$ control) was included for each master mix. All samples were 
Table I: Primers and probes

\begin{tabular}{ll}
\hline Sequence \\
\hline NGAL & \\
Forward primer & \\
Reverse primer & 5'-ATGACATGAACCTGCTCGATA-3' \\
Probe & 5'-TCATAGTCGTTCATTATCTTC-3' \\
MMP-9 & 5'-FAM- CCTGAAAAGAGTCTCTGCCCA-TAMRA-3' \\
Forward primer & \\
Reverse primer & 5'-CTGAAATGACGTCCCTAAGT-3' \\
Probe & 5'-AGGAGGTCTCACTATCTGGAT-3' \\
GAPDH & 5'-FAM- TCTGACATGCCTCGAGGACCT-TAMRA-3' \\
Forward primer & \\
Reverse primer & 5'-CCTCAAGATCATCAGCAAT-3' \\
Probe & 5'-CCATCCACAGTCTTCTGGGT-3' \\
\hline
\end{tabular}

amplified simultaneously in triplicate in a one-assay run. The amplified products were resolved by electrophoresis in $2.0 \%$ agarose gel and visualized by ethidium bromide staining. GAPDH was performed simultaneously as a standard internal control. The relative expression ratio (R) and real-time RT-PCR efficiency (E) of samples were determined by the following equations, as described in previous study[17,18].

$$
\begin{aligned}
& \mathrm{R}=\frac{\left(\mathrm{E}_{\text {target }}\right)^{\Delta \mathrm{CT}_{\text {target }}^{(\text {control-sample })}}}{\left(\mathrm{E}_{\text {reference }}\right)^{\Delta \mathrm{CT}}(\text { control-sample })}[17] \\
& \mathrm{E}=10^{-1 / \text { slope }}[18]
\end{aligned}
$$

The CT value is the cycle number at which the fluorescence signal crosses the threshold, and $\Delta \mathrm{CT}$ is the CT deviation of the control minus the sample of the target or reference gene transcript. A ratio $>1$ represented NGAL or MMP-9 mRNA up-regulation in rectal cancer relative to the control.

\section{Statistical analysis}

SPSS 13.0 software for Windows (SPSS Inc, USA) was used for statistical analysis. The relative expression analysis and group-wise comparison of the target gene was performed by the Relative Expression Software Tool (REST, available at http://www.gene-quantification.com)[19]. Continuous variables were expressed as $(\bar{x} \pm s)$. The expression of NGAL mRNA was assessed for associations with clinicopathological characteristics using Student's t-test for age and the $\chi^{2}$ test for the remaining parameters. Finally, the correlation between NGAL and MMP-9 was evaluated by calculating Spearman's correlation coefficient. $p<0.05$ was considered statistically significant.

\section{Results \\ NGAL and MMP-9 mRNA Expression in Rectal Cancer and Paired Normal Rectal Tissues}

The mRNA expression of NGAL and MMP-9 was examined in 100 rectal cancers and paired normal rectal tissues. Real-time RT-PCR amplification efficiencies were calculated from the given slopes in the FTC-2000 software (Fengling Co., Shanghai, China) and REST, and the amplification efficiency in the 2 groups was 2. As described above, the relative ratio, $R$, is presented as the nfold change in gene expression normalized to an endogenous reference gene and relative to the control. Therefore, a value of $\mathrm{R}>1.0$ was considered to represent over expression of NGAL and MMP-9 gene in the sample relative to the control.

Among the 100 rectal cancer RNA samples tested, NGAL and MMP-9 over expression were shown in 69 and 63 cases respectively. Over expression of mRNA NGAL and MMP-9 was observed as follows: 52 cases both over expressed, 20 cases both not over expressed, 17 cases NGAL over expressed but MMP-9 not overexpressed,11 cases MMP-9 over expressed but NGAL not over expressed. The numeric results of the randomization test were put in the randomization box. REST analysis showed mRNA up-regulation of NGAL and MMP-9 in the sample group was remarkably different from that in the control group ( $p=0.001)$, that is, it was up regulated in comparison to the control group (table 2, Figure 1).

\section{Relationship between NGAL mRNA up-regulation and Clinicopathological Features in Rectal Cancer}

The relationship between NGAL mRNA up-regulation and clinicopathological characteristics was analyzed. NGAL mRNA up-regulation correlated significantly with depth of invasion $(p=0.028)$, lymph node metastasis $(p=$ $0.009)$, venous involvement $(p=0.023)$ and advanced pTNM stage $(p=0.011)$. There was no correlation of 
Table 2: Output of randomisation analysis in $\mathbf{2}$ groups by REST

\begin{tabular}{lccc}
\hline & GAPDH & NGAL & MMP-9 \\
\hline Number & 100 & 100 & 100 \\
PCR efficiencies & 2 & 2 & 2 \\
Control means & 19.974 & 29.776 & 28.555 \\
Sample means & 20.077 & 27.364 & 26.889 \\
Expression ratios & & 5.716 & 3.408 \\
P values & & 0.001 & 0.001 \\
Expression ratios-nn & 0.931 & 5.322 & 3.173 \\
P values-nn & 0.709 & 0.001 & 0.001 \\
Randomisations & \multicolumn{3}{c}{$\mathrm{I}, 000$ of $\mathrm{I}, 000$ done } \\
\end{tabular}

REST: Relative expression software tool.

Sample means: Mean CT values for the rectal cancer tissues. Control means: Mean CT values for the adjacent normal tissues. Expression ratios-nn: Expression ratios not normalised. $p$ values-nn: $p$ values not normalised.

Randomisations: REST (Relative Expression Software Tool) is a standalone software tool to estimate up and down regulation for gene expression study in real-time PCR by using statistical randomization tests, while taking into account issues of reaction efficiency and reference gene normalisation. The randomization scenario is as follows: "if any perceived variation between samples and controls is due only to chance, then we could randomly swap values between the 2 groups and not see any greater difference than what we see between the initial groups."In our study, the hypothesis test performs I,000 random reallocations of samples and controls between the groups, and counts the number of times the relative expression on the randomly assigned group is greater than the sample data.

NGAL mRNA with age, gender and grade ( $p>0.05$, table 3).

\section{Correlation between mRNA up-regulation of NGAL and MMP-9 in Rectal Cancer}

In rectal cancers, the correlation coefficient between mRNA up-regulation of NGAL and MMP-9 was 0.393.
NGAL mRNA up-regulation was positively correlated with MMP-9 $(p<0.001$, table 4$)$.

\section{Discussion}

This is the first study to apply the real-time RT-PCR method to quantify the NGAL mRNA expression and to elucidate the correlation of NGAL mRNA expression with clinicopathologic features and MMP-9 in human rectal cancer. The present study observed that 69 cases (69\%) among the 100 rectal cancers showed NGAL mRNA overexpression, a significantly elevated level of NGAL mRNA expression with real-time RT-PCR in rectal cancers compared with adjacent normal tissues, which is in agreement with the report of Nielsen et al. [20] Hypomethylation of the NGAL gene has been found both in pancreatic and mammary tumor cell lines, which may be responsible for its high expression levels [21]. Moreover, we demonstrated that NGAL mRNA up-regulation correlated significantly with MMP-9 and some important clinicopathological characteristics such as depth of invasion, lymph node metastasis, venous involvement and advanced pTNM stage $(p<0.05)$. Depth of invasion, lymph node metastasis and pTNM stage of rectal cancer have powerful predictive value to prognosis. Venous invasion by tumor has also been demonstrated to be a stageindependent adverse prognostic factor $[22,23]$. This suggests that NGAL not only involved in the progression of rectal cancer, but also could be a prognostic marker in rectal cancer.

Interestingly, unlike our results, Lee and colleagues[24] reported that overexpression of NGAL suppressed human colon cancer KM12SM cell to invade Matrigel in vitro, and liver metastasis of colon cancer cells injected directly into the spleens in an mice experimental animal model. These

\section{Randomisation Data Output:}

Genes
PCR Efficiencies
Control Means
Sample Means
Expression Ratios
p-Values
Expression Ratios- $n n$
$p-$ Values- $n n$
Randomisations

Figure I

Output of randomisation analysis in 2 groups by REST. REST:Relative expression software tool. Genes ref.: GAPDH; Genes I: NGAL; Genes 2: MMP-9 Sample means: Mean CT values for the rectal cancer tissues. Control means:Mean CT values for the adjacent normal tissues. Expression ratios-nn: Expression ratios not normalised. $p$ values-nn: $p$ values not normalised. 
Table 3: Relationship between expression of NGAL mRNA and clinicopathological characteristics in rectal cancer

\begin{tabular}{|c|c|c|c|}
\hline \multirow[t]{2}{*}{ Variables/categories } & \multicolumn{2}{|c|}{ NGAL expression } & \multirow[t]{2}{*}{$P$ value } \\
\hline & ratio $\leq$ I & ratio > I & \\
\hline Age, years & $63.38 \pm 11.1$ & $60.78 \pm 13.1$ & NS \\
\hline Gender & & & NS \\
\hline Male & 13 & 31 & \\
\hline Female & 18 & 38 & \\
\hline Grade & & & NS \\
\hline Well-differentiated & 4 & 3 & \\
\hline Moderately differentiated & 20 & 51 & \\
\hline Poorly differentiated & 7 & 15 & \\
\hline Depth of invasion & & & 0.028 \\
\hline PTI & 5 & 2 & \\
\hline PT2 & 13 & 23 & \\
\hline PT3 & 12 & 33 & \\
\hline PT4 & 1 & 11 & \\
\hline Lymph node metastasis & & & 0.009 \\
\hline Absent & 17 & 19 & \\
\hline Present & 14 & 50 & \\
\hline Venous involvement & & & 0.023 \\
\hline Absent & 26 & 42 & \\
\hline Present & 5 & 27 & \\
\hline pTNM stage & & & 0.011 \\
\hline $\mathrm{pl}$ & 4 & 2 & \\
\hline pll & 13 & 14 & \\
\hline plll & 13 & 44 & \\
\hline pIV & 1 & 9 & \\
\hline
\end{tabular}

ratio $\leq \mathrm{I}$ : The expression of NGAL mRNA was not up-regulated; ratio > I: The expression of NGAL mRNA was up-regulated; NS:Not significant.

apparently diverse observations could be due to distinct functions of NGAL in different cell types, which is further supported by a previous study demonstrating that NGAL overexpression could suppress Ras-transformed murine breast cancer $4 \mathrm{~T} 1$ cell invasion and lung metastasis in vivo [25]. However, ectopic expression of NGAL may significantly stimulates the growth of MCF-7 human breast cancer cells in vivo[12]. Furthermore, such an experimental metastasis study may also have limitations in reflecting the whole process of metastasis since the effects on primary malignancy growth before metastasis could not be determined. Therefore, future evaluation of NGAL with a

Table 4: Correlation between mRNA up-regulation of NGAL and MMP-9

\begin{tabular}{ccccc}
\hline MMP-9 expression & \multicolumn{2}{c}{ NGAL expression } & $P$ & $\boldsymbol{r}$ \\
\cline { 2 - 3 } & ratio $\leq \mathrm{I}$ & ratio $>$ I & & \\
\hline ratio $\leq \mathrm{I}$ & 20 & 17 & $<0.001$ & 0.393 \\
ratio $>$ I & $1 \mathrm{I}$ & 52 & & \\
\hline
\end{tabular}

ratio $\leq 1$ : The expression of NGAL or MMP-9 mRNA was not upregulated; ratio > I: The expression of NGAL or MMP-9 mRNA was up-regulated; $r$ : Correlation coefficient. spontaneous metastasis assay was essential so as to elucidate the context-specific regulation mechanism.

MMPs are believed to play a crucial role in colorectal cancer tumor growth and angiogenesis, thereby promoting invasion and metastasis. MMP-9 appears to be one of the most important since it is overexpressed in the majority of colorectal cancers [26,27]. Several lines of evidence in vitro or in vivo have suggested that NGAL plays an important role in the enhanced invasive potential of cancer cells by upregulating MMP-9 expression. Yan et al demonstrated that NGAL is capable of protecting MMP-9 from degradation in a dose-dependent manner and thereby preserving MMP-9 enzymatic activity in vitro system by employing recombinant human MMP-9 and NGAL, as well as in a cell culture system in which NGAL was overexpressed in MDA-MB-231 human breast carcinoma cells. [11]Transfection of an NGAL expression plasmid conferred the invasive phenotype and motility activities of a noninvasive human breast cancer cell line (MCF-7) accompanying an increase in MMP-9 expression[12]. Furthermore, down-regulation of NGAL expression in oesophageal squamous cell carcinoma (ESCC) cells could significantly suppress MMP-9 activity and the invasion of these cells in nude mice[28]. Recently, Frank et al have reported the clinical evidence for a protective role of NGAL against MMP-9 autodegradation in gastric cancer[13]. In present study, we also revealed a significant elevated expression of NGAL and MMP-9 mRNA levels in human rectal cancer and they all correlated well with tumor invasion and metastasis. Additionally, NGAL mRNA overexpression was positively correlated with MMP-9 mRNA up-regulation ( $r s=0.393, p<0.001$ ). This suggests that the NGAL-MMP-9 axis could be a potent therapeutic target in rectal cancer patients. Therapeutic agents that inhibit the expression or function of NGAL or of its target genes may prove efficacious, or might complement agents that directly compromise the MMP-9 activities in the treatment of human rectal cancer. Studies are needed to further explore the mechanisms involved.

The spread of malignant tumor cells from a primary tumor to form metastases at distant sites is the most lifethreatening complication of cancer and is responsible for the majority of deaths in affected individuals. When detected at early stages, the majority of CRC can be cured with high success rates, and there is a strong correlation between tumor progression at the moment of the diagnosis and survival rate[29]. Hence, a marker to identify early stage cancer would be immensely useful to improve survival. For this purpose, a number of screening tests have been developed. However the ideal screening test must be specific, sensitive and non-invasive. NGAL as a valuable biomarker for early diagnosis and monitoring relapse of malignancy has been increasing used. Fernandez et al 
have demonstrated that NGAL and MMP-9 complexes were identified in nearly $86.36 \%$ of urine sample from breast cancer patients whereas this molecule is undetectable in the urine of healthy controls, suggesting that urinary NGAL may represent a novel, non-invasive biomarker for tracking disease status and the effectiveness of anticancer therapy in NGAL positive breast carcinomas[12]. In addition, serum NGAL measurement by ELISA is fairly accurate in distinguishing pancreatic cancer from non-cancer cases and could be investigated as a marker of pancreatic intraepithelial neoplasia (PIN) for early diagnosis of pancreatic cancer[30]. Furthermore, urinary NGAL and MMP-9 are useful predictors of the presence of brain tumors and may provide a basis for a novel, non-invasive method to identify new brain tumors and monitor known tumors after treatment[31]. Recently, Lim and colleagues[32] have also demonstrated that NGAL involved in the progression of epithelial ovarian malignanciesmay and may be served as an early screening biomarker to monitor changes of benign to premalignant and malignant ovarian tumors. This raises the possibility that serum and urine NGAL measurement (or combination with other potential biomarkers such as MMP-9) could also be explored as a utility for detecting disease status, progression, and therapeutic efficacy through noninvasive surveillance in rectal cancers, which deserves our attention.

\section{Conclusion}

Till now, little is known about the potential involvement of NGAL in human rectal cancer. In present study, we detected the mRNA expression of NGAL and MMP-9 in human rectal cancer by real-time RT-PCR. Our research demonstrated that NGAL mRNA expression was elevated in human rectal cancer. NGAL mRNA up-regulation was correlated significantly with tumor progression and MMP9 mRNA overexpression in rectal cancer, suggesting a more aggressive phenotype. NGAL detection may provide valuable information for rectal cancer characterization and identification of a subset of patients requiring more aggressive adjuvant therapy. As a secreted molecule, NGAL may serve as an attractive therapeutic target. Further studies are needed to elucidate the precise mechanisms of NGAL implicated in cancer progression and its potential utility in rectal cancer treatment.

\section{Competing interests}

The authors declare that they have no competing interests.

\section{Authors' contributions}

XFZ and SMZ carried out the experiments and drafted the manuscript. XHZ and GGY has been involved in revising the manuscript critically for important intellectual content. YZ participated in the design of the study and performed the statistical analysis. OCW participated in collecting the samples. GLG, GYY and XQH has been involved in designing the study and revising the manuscript. All authors read and approved the final manuscript.

\section{Acknowledgements}

We thank Chun-Yan Cai from the China National Optometry Research Center of Wenzhou Medical College for excellent technical assistance with real-time RT-PCR, and the colleagues of the Department of Oncological Surgery, the First Affiliated Hospital of Wenzhou Medical College, for providing experimental specimens. We also thank Prof. Guo-Rong Cheng from the Department of Pathology of the First Affiliated Hospital of Wenzhou Medical College for selfless assistance. This work was supported by grant from the National Natural Science Foundation of China, No. 30770487.

\section{References}

I. Boyle P, Ferlay J: Cancer incidence and mortality in Europe, 2004. Ann Oncol 2005, I 6(3):48I-488.

2. Jemal A, Siegel R, Ward E, Murray T, Xu J, Thun MJ: Cancer statistics, 2007. CA Cancer J Clin 2007, 57(I):43-66.

3. Aznavoorian S, Murphy AN, Stetler-Stevenson WG, Liotta LA: Molecular aspects of tumor cell invasion and metastasis. Cancer 1993, 7 I (4): I368- I383.

4. Poston G], Adam R, Alberts S, Curley S, Figueras J, Haller D, Kunstlinger F, Mentha G, Nordlinger B, Patt Y, et al:: OncoSurge: a strategy for improving resectability with curative intent in metastatic colorectal cancer. J Clin Oncol 2005, 23(28):7|25-7|34.

5. Stoesz SP, Gould MN: Overexpression of neu-related lipocalin (NRL) in neu-initiated but not ras or chemically initiated rat mammary carcinomas. Oncogene 1995, I I(I I):2233-224 I.

6. Kjeldsen L, Johnsen AH, Sengelov H, Borregaard $\mathrm{N}$ : Isolation and primary structure of NGAL, a novel protein associated with human neutrophil gelatinase. I Biol Chem 1993 268(14): 10425-10432.

7. Coles M, Diercks T, Muehlenweg B, Bartsch S, Zolzer V, Tschesche $\mathrm{H}$, Kessler $\mathrm{H}$ : The solution structure and dynamics of human neutrophil gelatinase-associated lipocalin. J Mol Biol 1999, 289(I):139-157.

8. Curran S, Murray GI: Matrix metalloproteinases in tumour invasion and metastasis. J Pathol 1999, 189(3):300-308.

9. Bergers G, Brekken R, McMahon G, Vu TH, Itoh T, Tamaki K, Tanzawa K, Thorpe P, Itohara S, Werb Z, et al.: Matrix metalloproteinase- 9 triggers the angiogenic switch during carcinogenesis. Nat Cell Biol 2000, 2( I 0):737-744.

10. Lochter A, Sternlicht MD, Werb Z, Bissell MJ: The significance of matrix metalloproteinases during early stages of tumor progression. Ann N Y Acad Sci 1998, 857: 180-193.

11. Yan L, Borregaard N, Kjeldsen L, Moses MA: The high molecular weight urinary matrix metalloproteinase (MMP) activity is a complex of gelatinase B/MMP-9 and neutrophil gelatinaseassociated lipocalin (NGAL). Modulation of MMP-9 activity by NGAL. J Biol Chem 200I, 276(40):37258-37265.

12. Fernandez CA, Yan L, Louis G, Yang J, Kutok JL, Moses MA: The matrix metalloproteinase-9/neutrophil gelatinase-associated lipocalin complex plays a role in breast tumor growth and is present in the urine of breast cancer patients. Clin Cancer Res 2005, I I ( I 5):5390-5395.

13. Kubben FJ, Sier CF, Hawinkels LJ, Tschesche H, van Duijn W, Zuidwijk K, Reijden JJ van der, Hanemaaijer R, Griffioen G, Lamers CB, et al.: Clinical evidence for a protective role of lipocalin-2 against MMP-9 autodegradation and the impact for gastric cancer. Eur J Cancer 2007, 43( (1 2): I 869- 1876.

14. Bauer M, Eickhoff JC, Gould MN, Mundhenke C, Maass N, Friedl A Neutrophil gelatinase-associated lipocalin (NGAL) is a predictor of poor prognosis in human primary breast cancer. Breast Cancer Res Treat 2008, I 08(3):389-397.

15. Zhang H, Xu L, Xiao D, Xie J, Zeng H, Wang Z, Zhang X, Niu Y, Shen $Z$, Shen J, et al.: Upregulation of neutrophil gelatinase-associated lipocalin in oesophageal squamous cell carcinoma: significant correlation with cell differentiation and tumour invasion. J Clin Pathol 2007, 60(5):555-561. 
16. Jass JR, Sobin LH: Histological typing of intestinal tumors; in: International Histological Classifications of Tumors. 2nd edition. Berlin, Springer-Verlag; 1989.

17. Pfaffl MW: A new mathematical model for relative quantification in real-time RT-PCR. Nucleic Acids Res 200I, 29(9):e45.

18. Bernard PS, Wittwer CT: Real-time PCR technology for cancer diagnostics. Clin Chem 2002, 48(8): I I78-I I85.

19. Pfaffl MW, Horgan GW, Dempfle L: Relative expression software tool (REST) for group-wise comparison and statistical analysis of relative expression results in real-time PCR. Nucleic Acids Res 2002, 30(9): 36 .

20. Nielsen BS, Borregaard N, Bundgaard JR, Timshel S, Sehested M, Kjeldsen L: Induction of NGAL synthesis in epithelial cells of human colorectal neoplasia and inflammatory bowel diseases. Gut 1996, 38(3):414-420.

21. Sato N, Fukushima N, Matsubayashi H, Goggins M: Identification of maspin and SI OOP as novel hypomethylation targets in pancreatic cancer using global gene expression profiling. Oncogene 2004, 23(8): $153 \mid-1538$.

22. Knudsen JB, Nilsson T, Sprechler M, Johansen A, Christensen N: Venous and nerve invasion as prognostic factors in postoperative survival of patients with resectable cancer of the rectum. Dis Colon Rectum 1983, 26(9):6|3-6I7.

23. Horn $A$, Dahl $O$, Morild I: Venous and neural invasion as predictors of recurrence in rectal adenocarcinoma. Dis Colon Rectum |99|, 34(9):798-804.

24. Lee HJ, Lee EK, Lee KJ, Hong SW, Yoon Y, Kim JS: Ectopic expression of neutrophil gelatinase-associated lipocalin suppresses the invasion and liver metastasis of colon cancer cells. Int J Cancer 2006, I I 8( I 0):2490-2497.

25. Hanai J, Mammoto T, Seth P, Mori K, Karumanchi SA, Barasch J, Sukhatme VP: Lipocalin 2 diminishes invasiveness and metastasis of Ras-transformed cells. J Biol Chem 2005, 280( | 4): | 364|-|3647.

26. Nagase $\mathrm{H}$, Woessner JF: Matrix metalloproteinases. J Biol Chem 1999, 274(3I):2।49|-2।494.

27. Stetler-Stevenson WG: Matrix metalloproteinases in angiogenesis: a moving target for therapeutic intervention. J Clin Invest 1999, I03(9): I237-124I.

28. Li EM, Xu LY, Cai WJ, Xiong HQ, Shen ZY, Zeng Y: Functions of neutrophil gelatinase-associated lipocalin in the esophageal carcinoma cell line SHEEC. Sheng Wu Hua Xue Yu Sheng Wu Wu Li Xue Bao (Shanghai) 2003, 35(3):247-254.

29. Davies RJ, Miller R, Coleman N: Colorectal cancer screening: prospects for molecular stool analysis. Nat Rev Cancer 2005 5(3): 199-209.

30. Moniaux N, Chakraborty S, Yalniz M, Gonzalez J, Shostrom VK, Standop J, Lele SM, Ouellette M, Pour PM, Sasson AR, et al.: Early diagnosis of pancreatic cancer: neutrophil gelatinase-associated lipocalin as a marker of pancreatic intraepithelial neoplasia. Br J Cancer 2008, 98(9): I540-I547.

31. Smith ER, Zurakowski D, Saad A, Scott RM, Moses MA: Urinary biomarkers predict brain tumor presence and response to therapy. Clin Cancer Res 2008, 14(8):2378-2386.

32. Lim R, Ahmed N, Borregaard N, Riley C, Wafai R, Thompson EW, Quinn MA, Rice GE: Neutrophil gelatinase-associated lipocalin (NGAL) an early-screening biomarker for ovarian cancer: NGAL is associated with epidermal growth factor-induced epithelio-mesenchymal transition. Int J Cancer 2007, I 20(I I):2426-2434.

\section{Pre-publication history}

The pre-publication history for this paper can be accessed here:

\section{http://www.biomedcentral.com/1471-2407/9/134/pre} pub
Publish with Biomed Central and every scientist can read your work free of charge

"BioMed Central will be the most significant development for disseminating the results of biomedical research in our lifetime. "

Sir Paul Nurse, Cancer Research UK

Your research papers will be:

- available free of charge to the entire biomedical community

- peer reviewed and published immediately upon acceptance

- cited in PubMed and archived on PubMed Central

- yours - you keep the copyright
BioMedcentral 